\title{
A Field Emission Scanning Electron Microscopy Method to Assess Recombinant Adenovirus Stability.
}

\author{
L. J. Obenauer-Kutner, ${ }^{*}$ P. M. Ihnat, ${ }^{* *}$ T-Y. Yang, ${ }^{*}$ B. J. Dovey-Hartman, ${ }^{* * *}$ and M. J. Grace.* \\ *Schering-Plough Research Institute (SPRI), Biotechnology Development, Union, NJ 07083 \\ **SPRI, Pharmaceutical Development, Kenilworth, NJ 07033 \\ ***SPRI, Drug Safety and Metabolism, Lafayette, NJ 07848
}

A field emission scanning electron microscopy (FESEM) method was developed to assess recombinant adenovirus ( $\mathrm{rAd} / \mathrm{p} 53$ ) stability. This method was designed to simultaneously sort, count, and size the total number of $\mathrm{rAd} / \mathrm{p} 53$ objects observed in an image. To test the method we treated a $\mathrm{rAd} / \mathrm{p} 53$ preparation $[1,5]$ with thermal incubation at $37^{\circ} \mathrm{C}$ for $0,0.5,1.0,1.5,2.0,3.0$, and 4.0 hours, and then monitored the effect on stability by assessing the anatomy of the virions using FESEM, with automated image-analysis (Image-Pro Plus v.4.1, Media Cybernetics, Silver Spring, $\mathrm{MD}$ ), and transmission electron microscopy (TEM). In addition to electron microscopy (EM), the infectious activity of the thermally stressed $\mathrm{rAd} / \mathrm{p} 53$ samples was quantitated using an established flow cytometry method. [2,3]

Unconjugated gold particles were mixed with each sample post-incubation to facilitate focusing and to provide an internal control for sizing of the virus particles. Viral specimens were subsequently fixed and stained on carbon coated copper grids, and then prepared for EM image-analysis. The T=0 time point showed that virions were evenly distributed over the grid, and the icosahedral geometry of the virus was evident. [4] As shown in Figure 1, FESEM image-analysis revealed a decrease in the total number of detectable single $\mathrm{rAd} / \mathrm{p} 53$ particles and an increase in apparent micro-aggregates composed of multiple viral particles (multiplets) as early as 2 hours. In addition there was an observed decrease in the size of the single $\mathrm{rAd} / \mathrm{p} 53$ particles and an increase in multiplet size with time at $37^{\circ} \mathrm{C}$. The described changes predominated after 4 hours of incubation. The changes noted in virus morphology were concomitant with the observed loss in viral infectivity (Figure 2). The FESEM results were reproduced with TEM.

In conclusion, the results reported in this study suggest a novel method of assessing the stability of recombinant adenovirus products using FESEM image-analysis. The decrease in single $\mathrm{rAd} / \mathrm{p} 53$ particles and the increase in higher order multiplets suggest that the method may be useful for monitoring or assessing stability.

References

[1] B. Huyghe, et al., Human Gene Therapy. 6 (1995) 1403.

[2] M. L. Musco, et al., Cytometry. 33, (1998) 290.

[3] C. Nyberg-Hoffman, et al., Nature Medicine. 3 (1997) 808.

[4] L. Philipson, Curr. Top. Microbiol. Immunol. 109 (1983) 1.

[5] P. W. Shabram, et al., Human Gene Therapy. 8 (1997) 453. 
(a)

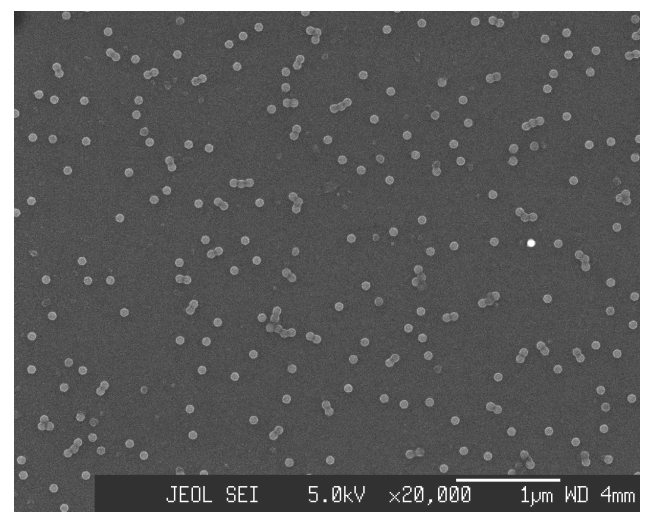

(c)

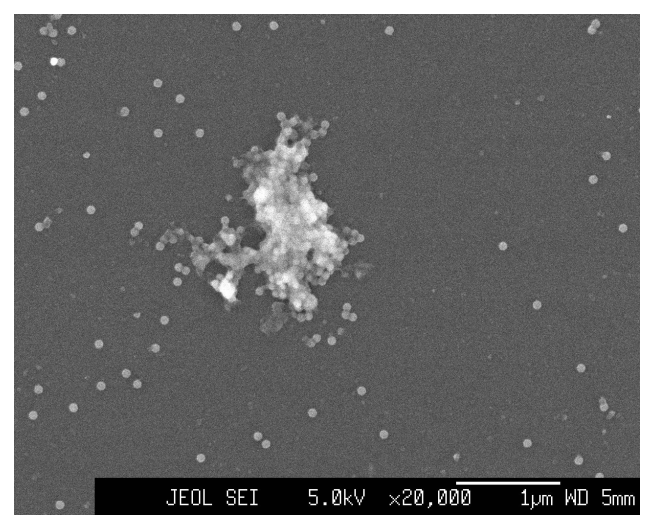

(b)

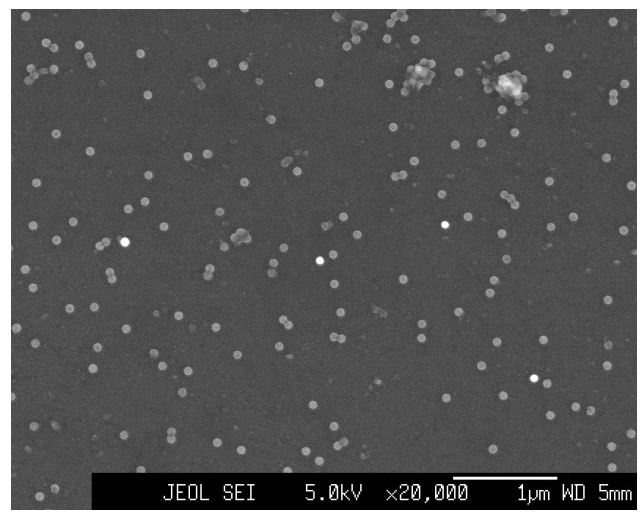

(d)

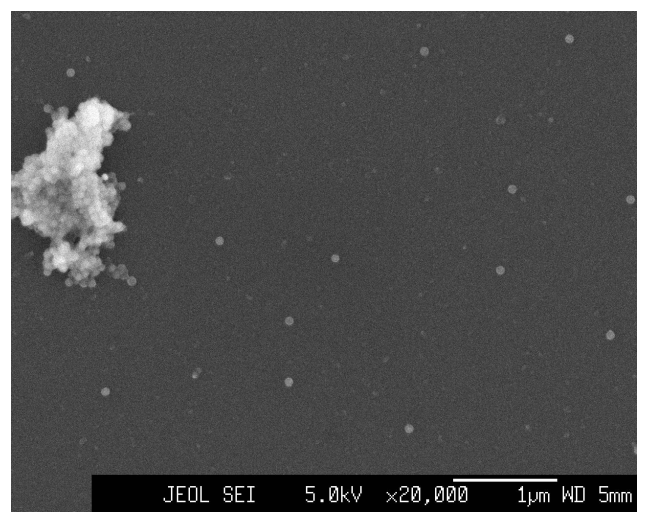

FIG.1. Representative FESEM images of $\mathrm{rAd} / \mathrm{p} 53$ in PBS incubated $a 7^{\circ} \mathrm{C}$ at the various times indicated. (a) $\mathrm{T}=0$ (b) $\mathrm{T}=0.5$ hours (c) $\mathrm{T}=2.0$ hours (d) $\mathrm{T}=4.0$ hours

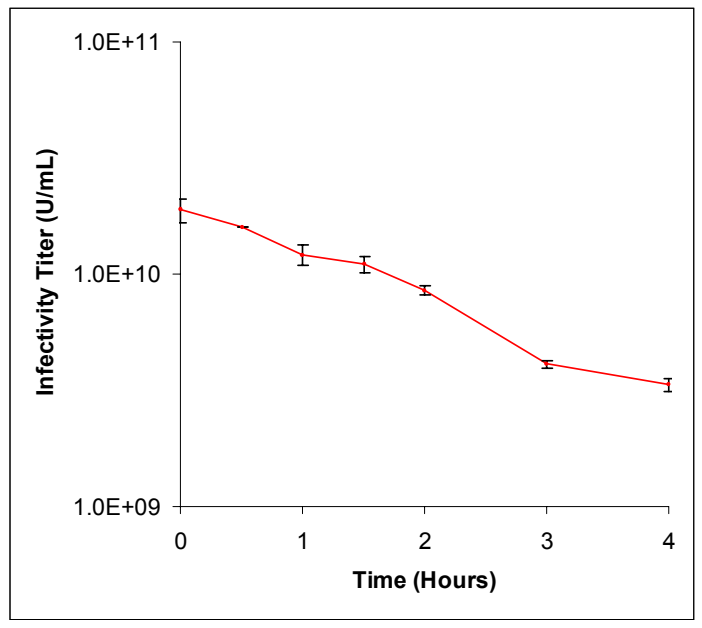

FIG. 2. Representative plot showing kinetics of $\mathrm{rAd} / \mathrm{p} 53$ infectivity during thermal stress at $37^{\circ} \mathrm{C}$. 